\title{
On growth of the set $A(A+1)$ in arbitrary finite fields
}

\author{
Ali Mohammadi \\ School of Mathematics \\ Institute for Research in Fundamental Sciences (IPM) \\ Tehran, Iran \\ a.mohammadi@ipm.ir
}

Submitted: Feb 3, 2019; Accepted: Sep 8, 2020; Published: Oct 2, 2020

(C) The author. Released under the CC BY-ND license (International 4.0).

\begin{abstract}
Let $\mathbb{F}_{q}$ be a finite field of order $q$, where $q$ is a power of a prime. For a set $A \subset \mathbb{F}_{q}$, under certain structural restrictions, we prove a new explicit lower bound on the size of the product set $A(A+1)$. Our result improves on the previous best known bound due to Zhelezov and holds under more relaxed restrictions.
\end{abstract}

Mathematics Subject Classifications: 11B75

\section{Introduction}

Let $p$ denote a prime, $\mathbb{F}_{q}$ the finite field consisting of $q=p^{m}$ elements and $\mathbb{F}_{q}^{*}=\mathbb{F}_{q} \backslash\{0\}$. For sets $A, B \subset \mathbb{F}_{q}$, we define the sum set $A+B=\{a+b: a \in A, b \in B\}$ and the product set $A B=\{a b: a \in A, b \in B\}$. Similarly, we define the difference set $A-B$ and the ratio set $A / B$.

The sum-product phenomenon in finite fields is the assertion that for $A \subset \mathbb{F}_{q}$, the sets $A+A$ and $A A$ cannot both simultaneously be small unless $A$ closely correlates with a coset of a subfield. A result in this direction is due to Li and Roche-Newton [6], who showed that if $|A \cap c G| \leqslant|G|^{1 / 2}$ for all subfields $G$ and elements $c$ in $\mathbb{F}_{q}$, then

$$
\max \{|A+A|,|A A|\} \gg(\log |A|)^{-5 / 11}|A|^{1+1 / 11} .
$$

In the same spirit and under a similar structural assumption on the set $A$, one expects that, for all $\alpha \in \mathbb{F}_{q}^{*}$, either of the product sets $A A$ or $(A+\alpha)(A+\alpha)$ must be significantly larger than $A$. Zhelezov [12] proved the estimate

$$
\max \{|A B|,|(A+1) C|\} \gtrsim|A|^{1+1 / 559}
$$


for sets $A, B, C \subset \mathbb{F}_{q}$, under the condition that

$$
|A B \cap c G| \leqslant|G|^{1 / 2}
$$

for all subfields $G$ of $\mathbb{F}_{q}$ and elements $c \in \mathbb{F}_{q}$. Then, taking $B=A$ and $C=A+1$, under restriction (2), we have

$$
\max \{|A A|,|(A+1)(A+1)|\} \gtrsim|A|^{1+1 / 559} .
$$

For sets $B_{1}, B_{2}, X \subset \mathbb{F}_{q}^{*}$, we recall Plünnecke's inequality (see Lemma 9)

$$
\left|B_{1} B_{2}\right| \leqslant \frac{\left|B_{1} X\right|\left|B_{2} X\right|}{|X|} .
$$

From this we can deduce that

$$
|A(A+1)|^{2} \geqslant|A| \cdot \max \{|A A|,|(A+1)(A+1)|\} .
$$

Hence, by (3), we have the estimate

$$
|A(A+1)| \gtrsim|A|^{1+\delta}
$$

with $\delta=1 / 1118$, which holds under restriction (2) with $B=A$. Alternatively, by (1), with $B=A+1$ and $C=A$, the estimate (4) holds with $\delta=1 / 559$.

For large sets, $A \subset \mathbb{F}_{q}$ with $|A| \geqslant q^{1 / 2}$, Garaev and Shen [2] proved the bound

$$
|A(A+1)| \gg \min \left\{q^{1 / 2}|A|^{1 / 2},|A|^{2} / q^{1 / 2}\right\} .
$$

Furthermore, it was demonstrated in [2] that in the range $|A|>q^{2 / 3}$, the bound (5) is optimal up to the implied constant.

In the realm of small sets $A \subset \mathbb{F}_{q}$, with $|A| \leqslant p^{5 / 8}$, Stevens and de Zeeuw [9] obtained

$$
|A(A+1)| \gg|A|^{1+1 / 5} .
$$

Warren [11], further improved this bound to $(\log |A|)^{-7 / 6}|A|^{1+2 / 9}$ under the constraint $|A| \leqslant p^{1 / 4}$. Both of these results are based on a bound on incidences between lines and Cartesian products, proved in [9], which in turn relies on a bound on incidences between points and planes due to Rudnev [8]. We point out that the main result of [8] has led to many quantitatively strong sum-product type estimates, however these estimates are restricted to sets which are bounded in size in terms of the characteristic $p$.

Our main result, stated below, relies on a somewhat more primitive approach towards the sum-product problem in finite fields, often referred to as the additive pivot technique. Specifically, we adopt our main tools and ideas from [4] and [6].

Theorem 1. Let $A \subseteq \mathbb{F}_{q}$. Suppose that

$$
|A \cap c G| \ll \max \left\{|G|^{1 / 2},|A|^{25 / 26}\right\}
$$

for all proper subfields $G$ of $\mathbb{F}_{q}$ and elements $c \in \mathbb{F}_{q}$. Then for all $\alpha \in \mathbb{F}_{q}^{*}$, we have

$$
|A(A+\alpha)| \gtrsim \min \left\{|A|^{1+1 / 52}, q^{1 / 48}|A|^{1-1 / 48}\right\} .
$$


Theorem 1 provides a quantitative improvement over the relevant estimates implied by (1) and holds under a more relaxed condition than those given by (2). It also improves on (5) in the range $q^{1 / 2} \leqslant|A| \lesssim q^{1 / 2+1 / 102}$.

Given a set $A \subset \mathbb{F}_{q}$, we define the additive energy of $A$ as the quantity

$$
E_{+}(A)=\left|\left\{\left(a_{1}, a_{2}, a_{3}, a_{4}\right) \in A^{4}: a_{1}+a_{2}=a_{3}+a_{4}\right\}\right| .
$$

As an application of Theorem 1, we give a bound on the additive energy of subsets of $\mathbb{F}_{q}$.

Corollary 2. Let $A \subseteq \mathbb{F}_{q}$. Suppose that

$$
|A \cap c G| \ll \max \left\{|G|^{1 / 2},|A A|^{50 / 53}\right\}
$$

for all proper subfields $G$ of $\mathbb{F}_{q}$ and elements $c \in \mathbb{F}_{q}$. Then for any $\alpha \in \mathbb{F}_{q}^{*}$, we have

$$
|A \cap(A-\alpha)| \lesssim|A A|^{1-1 / 53}+q^{-1 / 47}|A A|^{1+1 / 47}
$$

Consequently, under restriction (7), we have

$$
E_{+}(A) \lesssim|A|^{2}\left(|A A|^{1-1 / 53}+q^{-1 / 47}|A A|^{1+1 / 47}\right) .
$$

\section{Asymptotic notation}

We use standard asymptotic notation. In particular, for positive real numbers $X$ and $Y$, we use $X=O(Y)$ or $X \ll Y$ to denote the existence of an absolute constant $c>0$ such that $X \leqslant c Y$. If $X \ll Y$ and $Y \ll X$, we write $X=\Theta(Y)$ or $X \approx Y$. We also use $X \lesssim Y$ to denote the existence of an absolute constant $c>0$, such that $X \ll(\log Y)^{c} Y$.

\section{Preparations}

For $X \subset \mathbb{F}_{q}$, let $R(X)$ denote the quotient set of $X$, defined by

$$
R(X)=\left\{\frac{x_{1}-x_{2}}{x_{3}-x_{4}}: x_{1}, x_{2}, x_{3}, x_{4} \in X, x_{3} \neq x_{4}\right\} .
$$

We present a basic extension of [10, Lemma 2.50].

Lemma 3. Let $X \subset \mathbb{F}_{q}$ and $r \in \mathbb{F}_{q}^{*}$. If $r \notin R(X)$, for any nonempty subsets $X_{1}, X_{2} \subseteq X$, we have

$$
\left|X_{1}\right|\left|X_{2}\right|=\left|X_{1}-r X_{2}\right| \text {. }
$$

Proof. Consider the mapping $\phi: X_{1} \times X_{2} \rightarrow X_{1}-r X_{2}$ defined by $\phi\left(x_{1}, x_{2}\right)=x_{1}-r x_{2}$. Suppose that $\left(x_{1}, x_{2}\right),\left(y_{1}, y_{2}\right) \in X_{1} \times X_{2}$ are distinct pairs satisfying $x_{1}-r x_{2}=y_{1}-r y_{2}$. Then we get

$$
r=\frac{x_{1}-y_{1}}{x_{2}-y_{2}}
$$

which contradicts the assumption that $r \notin R(X)$. We deduce that $\phi$ is injective, which in turn implies the required result. 
The next lemma, which appeared in [10, Corollary 2.51], is a simple corollary of Lemma 3.

Lemma 4. Let $X \subset \mathbb{F}_{q}$ with $|X|>q^{1 / 2}$, then $R(X)=\mathbb{F}_{q}$.

We have extracted Lemma 5, stated below, from the proof of the main result in [6].

Lemma 5. Let $X \subset \mathbb{F}_{q}$ be such that

$$
1+R(X) \subseteq R(X) \text { and } X \cdot R(X) \subseteq R(X) .
$$

Then $R(X)$ is the subfield of $\mathbb{F}_{q}$ generated by $X$.

The next result has been stated and proved in the proof of [7, Theorem 1].

Lemma 6. Let $X \subset \mathbb{F}_{q}$ with $|R(X)| \gg|X|^{2}$. Then there exists $r \in R(X)$ such that for any subset $X^{\prime} \subset X$ with $\left|X^{\prime}\right| \approx|X|$, we have

$$
\left|X^{\prime}+r X^{\prime}\right| \gg|X|^{2}
$$

The following lemma enables us to extend our main result to sets which are larger than $q^{1 / 2}$. See [1, Lemma 3] for a proof.

Lemma 7. Let $X_{1}, X_{2} \subset \mathbb{F}_{q}$. There exists an element $\xi \in \mathbb{F}_{q}^{*}$ such that

$$
\left|X_{1}+\xi X_{2}\right| \geqslant \frac{\left|X_{1}\right|\left|X_{2}\right|(q-1)}{\left|X_{1}\right|\left|X_{2}\right|+(q-1)} .
$$

Next, we recall Ruzsa's triangle inequality. See [10, Lemma 2.6] for a proof.

Lemma 8. Let $X, B_{1}, B_{2}$ be nonempty subsets of an abelian group. We have

$$
\left|B_{1}-B_{2}\right| \leqslant \frac{\left|X+B_{1}\right|\left|X+B_{2}\right|}{|X|} .
$$

In particular, for $A \subset \mathbb{F}_{q}^{*}$, by a multiplicative application of Lemma 8, we have the useful inequality

$$
|A / A| \leqslant \frac{|A(A+1)|^{2}}{|A|} .
$$

In the next two lemmas we state variants of the Plünnecke-Ruzsa inequality, which can also be found in [5].

Lemma 9. Let $X, B_{1}, \ldots, B_{k}$ be nonempty subsets of an abelian group. Then

$$
\left|B_{1}+\cdots+B_{k}\right| \leqslant \frac{\left|X+B_{1}\right| \cdots\left|X+B_{k}\right|}{|X|^{k-1}} .
$$


Lemma 10. Let $X, B_{1}, \ldots, B_{k}$ be nonempty subsets of an abelian group. For any $0<$ $\epsilon<1$, there exists a subset $X^{\prime} \subseteq X$, with $\left|X^{\prime}\right| \geqslant(1-\epsilon)|X|$ such that

$$
\left|X^{\prime}+B_{1}+\cdots+B_{k}\right| \ll_{\epsilon, k} \frac{\left|X+B_{1}\right| \cdots\left|X+B_{k}\right|}{|X|^{k-1}} .
$$

The following two lemmas are due to Jones and Roche-Newton [4].

Lemma 11. Let $Z \subseteq \mathbb{F}_{q}^{*}$. Suppose that $X, Y \subseteq x Z+y$ for some $x \in \mathbb{F}_{q}^{*}$ and $y \in \mathbb{F}_{q}$. Fix $0<\epsilon<1 / 16$. Then, $(1-\epsilon)|X|$ elements of $X$ can be covered by

$$
O_{\epsilon}\left(\frac{|Z(Z+1)|^{2}|Z / Z|}{|X||Y|^{2}}\right)
$$

translates of $Y$. Similarly, $(1-\epsilon)|X|$ elements of $X$ can be covered by this many translates of $-Y$.

Lemma 12. Let $A \subseteq \mathbb{F}_{q}^{*}$. There exists a subset $A^{\prime} \subseteq A$ with $\left|A^{\prime}\right| \approx|A|$ such that

$$
\left|A^{\prime}-A^{\prime}\right| \ll \frac{|A(A+1)|^{4}|A / A|^{2}}{|A|^{5}} .
$$

Next, we record a popularity pigeonholing argument. A proof is provided in [3, Lemma 9].

Lemma 13. Let $X$ be a finite set and let $f$ be a function such that $f(x)>0$ for all $x \in X$. Suppose that

$$
\sum_{x \in X} f(x) \geqslant K
$$

Let $Y=\{x \in X: f(x) \geqslant K / 2|X|\}$. Then

$$
\sum_{y \in Y} f(y) \geqslant \frac{K}{2} .
$$

Additionally, if $f(x) \leqslant M$ for all $x \in X$, then $|Y| \geqslant K /(2 M)$.

For sets $X, Y \subseteq \mathbb{F}_{q}$, we define the multiplicative energy between $X$ and $Y$ as the quantity

$$
E_{\times}(X, Y)=\left|\left\{\left(x_{1}, x_{2}, y_{1}, y_{2}\right) \in X^{2} \times Y^{2}: x_{1} y_{1}=x_{2} y_{2}\right\}\right|
$$

and write simply $E_{\times}(X)$ instead of $E_{\times}(X, X)$. For $\xi \in Y / X$, let

$$
r_{Y: X}(\xi)=|\{(x, y) \in X \times Y: y / x=\xi\}| .
$$

Then, we have the identities

$$
\sum_{\xi \in Y / X} r_{Y: X}(\xi)=|X||Y|
$$




$$
\sum_{\xi \in Y / X} r_{Y: X}^{2}(\xi)=E_{\times}(X, Y)
$$

By a simple application of the Cauchy-Schwarz inequality we have

$$
E_{\times}(X, Y)|X Y| \geqslant|X|^{2}|Y|^{2} \text {. }
$$

The remaining two lemmas together form the basis for the proof of Theorem 1 . Lemma 15 is a slight generalisation of [7, Lemma 3].

Lemma 14. Let $X, Y \subset \mathbb{F}_{q}$, with $|Y| \leqslant|X|$. There exists a set $D \subseteq Y / X$ and an integer $N \leqslant|Y|$ such that $E_{\times}(X, Y) \ll(\log |X|)|D| N^{2}$ and $|D| N<|X||Y|$. Also, for $\xi \in D$ we have $r_{Y: X}(\xi) \approx N$. Namely, the set of points

$$
P=\{(x, y) \in X \times Y: y / x \in D\}
$$

is supported on $|D|$ lines through the origin, with each line containing $\Theta(N)$ points of $P$. Proof. For $j \geqslant 0$, let $L_{j}=\left\{\xi \in Y / X: 2^{j} \leqslant r_{Y: X}(\xi)<2^{j+1}\right\}$. Then, by (11), we have

$$
\sum_{j=0}^{\left\lfloor\log _{2}|X|\right\rfloor} \sum_{\xi \in L_{j}} r_{Y: X}^{2}(\xi)=E_{\times}(X, Y) .
$$

By the pigeonhole principle there exists some $N \geqslant 1$ such that, letting $D=\{\xi \in Y / X$ : $\left.N \leqslant r_{Y: X}(\xi)<2 N\right\}$, we have

$$
\frac{E_{\times}(X, Y)}{\log |X|} \ll \sum_{\xi \in D} r_{Y: X}^{2}(\xi) \ll|D| N^{2} .
$$

Furthermore, by (10), we have

$$
|D| N<\sum_{\xi \in D} r_{Y: X}(\xi) \leqslant|X||Y| .
$$

Lemma 15. Let $X, Y \subset \mathbb{F}_{q}$. Suppose $P \subset X \times Y$ is a set of points supported on $L$ lines through the origin, with each line containing $\Theta(N)$ points of $P$, so that $|P| \approx L N$. For $x_{*} \in X$ and $y_{*} \in Y$, we write $Y_{x_{*}}=\left\{y \in Y:\left(x_{*}, y\right) \in P\right\}$ and $X_{y_{*}}=\left\{x \in X:\left(x, y_{*}\right) \in\right.$ $P\}$. There exists a popular abscissa $x_{0}$ and a popular ordinate $y_{0}$, so that

$$
\left|Y_{x_{0}}\right| \gg \frac{L N}{|X|}, \quad\left|X_{y_{0}}\right| \gg \frac{L N}{|Y|}
$$

For $\xi \in \mathbb{F}_{q}$, we write $P_{\xi}=\{x:(x, \xi x) \in P\}$. There exists a subset $\widetilde{Y}_{x_{0}} \subseteq Y_{x_{0}}$ with

$$
\left|\widetilde{Y}_{x_{0}}\right| \gg \frac{L^{2} N^{2}}{|X|^{2}|Y|}
$$

such that for every $z \in \widetilde{Y}_{x_{0}}$, we have

$$
\left|P_{z / x_{0}} \cap X_{y_{0}}\right| \gg \frac{L^{2} N^{3}}{|X|^{2}|Y|^{2}} .
$$


Proof. Observing that

$$
\sum_{y \in Y}\left|X_{y}\right|=|P| \approx L N
$$

by Lemma 13, there exists a subset $Y^{\prime} \subseteq Y$ such that, for all $y \in Y^{\prime}$, we have $\left|X_{y}\right| \gg$ $L N /|Y|$. Let $P^{\prime}=\left\{(x, y) \in P: y \in Y^{\prime}\right\}$ so that $\left|P^{\prime}\right| \gg L N$. Then

$$
\sum_{x \in X}\left|Y_{x} \cap Y^{\prime}\right|=\sum_{y \in Y^{\prime}}\left|X_{y}\right|=\left|P^{\prime}\right| \gg L N
$$

By Lemma 13, there exists a subset $X^{\prime} \subseteq X$ such that for all $x \in X^{\prime}$ we have

$$
\left|Y_{x} \cap Y^{\prime}\right| \gg \frac{L N}{|X|}
$$

Letting $P^{\prime \prime}=\left\{(x, y) \in P^{\prime}: x \in X^{\prime}\right\}$, we have $\left|P^{\prime \prime}\right| \gg L N$.

Let $D=\left\{y / x:(x, y) \in P^{\prime \prime}\right\}$ and let $D^{\prime} \subseteq D$ denote the set of elements $\xi$ such that the lines $l_{\xi}$, determined by $\xi$, each contain $\Omega(N)$ points of $P^{\prime \prime}$. It follows by Lemma 13 that $\left|D^{\prime}\right| \gg L$. Now, we proceed to establish a lower bound on the sum

$$
\Sigma=\sum_{(x, y) \in X^{\prime} \times Y^{\prime}} \sum_{z \in Y_{x}}\left|P_{z / x} \cap X_{y}\right| .
$$

We write $z \sim x$, if $(x, z)$ is a point of $P$. Then

$$
\begin{aligned}
\Sigma & \sum_{\substack{(x, y) \in X^{\prime} \times Y^{\prime} \\
z: z \sim x}}\left|P_{z / x} \cap X_{y}\right| \\
& \gg N \sum_{\xi \in D^{\prime}} \sum_{y \in Y^{\prime}}\left|P_{\xi}^{\prime \prime} \cap X_{y}\right| .
\end{aligned}
$$

For a fixed $\xi \in D^{\prime}$, the inner sum may be bounded by the observation that

$$
\sum_{y \in Y^{\prime}}\left|P_{\xi}^{\prime \prime} \cap X_{y}\right|=\sum_{x \in P_{\xi}^{\prime \prime}}\left|Y_{x} \cap Y^{\prime}\right| .
$$

Recall that $\left|D^{\prime}\right| \gg L$ and that for $\xi \in D^{\prime}$, we have $\left|P_{\xi}^{\prime \prime}\right| \gg N$. Then, by (15), we have

$$
\Sigma \gg N \cdot L \cdot N \cdot \frac{L N}{|X|}
$$

By the pigeonhole principle, applied to (16), there exist $\left(x_{0}, y_{0}\right) \in X^{\prime} \times Y^{\prime}$ such that

$$
\sum_{z \in Y_{x_{0}}}\left|P_{z / x_{0}} \cap X_{y_{0}}\right| \gg \frac{L^{2} N^{3}}{|X|^{2}|Y|} .
$$


By our assumption, that every line through the origin contains $O(N)$ points of $P$, it follows that for all $z \in Y$, we have $\left|P_{z / x_{0}}\right| \ll N$. Then, letting $\widetilde{Y}_{x_{0}} \subseteq Y_{x_{0}}$ to denote the set of $z \in Y_{x_{0}}$ with the property that

$$
\left|P_{z / x_{0}} \cap X_{y_{0}}\right| \gg \frac{L^{2} N^{3}}{|X|^{2}|Y|^{2}}
$$

by Lemma 13, we have

$$
\left|\widetilde{Y}_{x_{0}}\right| \gg \frac{L^{2} N^{2}}{|X|^{2}|Y|}
$$

\section{Proof of Theorem 1}

It suffices to prove the required result for $\alpha=1$. Then the general statement immediately follows since under condition (6) the set $A$ can be replaced by any of its dilates $c A$, for $c \in \mathbb{F}_{q}^{*}$. Without loss of generality assume $0 \notin A$. By Lemma 12, combined with (9), there exists a subset $A^{\prime} \subseteq A$, with $\left|A^{\prime}\right| \approx|A|$, such that

$$
\left|A^{\prime}-A^{\prime}\right| \ll \frac{|A(A+1)|^{8}}{|A|^{7}} .
$$

By Lemma 10 there exists a further subset $A^{\prime \prime} \subseteq A^{\prime}$, with $\left|A^{\prime \prime}\right| \approx\left|A^{\prime}\right|$, such that

$$
\left|A^{\prime \prime}-A^{\prime \prime}-A^{\prime \prime}-A^{\prime \prime}\right| \ll \frac{\left|A^{\prime}-A^{\prime}\right|^{3}}{|A|^{2}} .
$$

Since $\left|A^{\prime \prime}\right| \approx|A|$, we reset the notation $A^{\prime \prime}$ back to $A$ and henceforth assume the inequalities

$$
\begin{gathered}
|A-A| \ll \frac{|A(A+1)|^{8}}{|A|^{7}}, \\
|A-A-A-A| \ll \frac{|A(A+1)|^{24}}{|A|^{23}} .
\end{gathered}
$$

We apply Lemma 14 to identify a set $D \subseteq A /(A+1)$ and an integer $N \geqslant 1$ such that for $\xi \in D$ we have $r_{A:(A+1)}(\xi) \approx N$. Additionally, letting $L=|D|$, in view of $(12)$, we have

$$
M:=L N^{2} \gg \frac{E_{\times}(A+1, A)}{\log |A|} \geqslant \frac{|A|^{4}}{|A(A+1)| \log |A|} .
$$

We define $P \subseteq(A+1) \times A$ by

$$
P=\{(x, y) \in(A+1) \times A: y / x \in D\} .
$$

Then $|P| \approx L N$. Now, since $L N<|A|^{2}$ and $N<|A|$, we get

$$
N, L>\frac{M}{|A|^{2}} \text {. }
$$


For $\xi \in D$, we define the projection onto the $x$-axis of the line with slope $\xi$ as

$$
P_{\xi}=\{x:(x, \xi x) \in P\} \subset A+1 .
$$

Similarly for $\lambda \in D^{-1}$ let

$$
Q_{\lambda}=\{y:(\lambda y, y) \in P\} \subset A .
$$

Then for $\xi \in D$ and $\lambda \in D^{-1}$, we have

$$
\left|P_{\xi}\right|,\left|Q_{\lambda}\right| \approx N, \quad \xi P_{\xi} \subset A \text { and } \lambda Q_{\lambda} \subset A+1 .
$$

By Lemma 15, with $X=A+1$ and $Y=A$, there exists a pair of elements $\left(x_{0}, y_{0}\right) \in$ $(A+1) \times A$ such that the sets $A_{x_{0}} \subseteq A$ and $B_{y_{0}} \subseteq A+1$ satisfy

$$
\left|A_{x_{0}}\right|,\left|B_{y_{0}}\right| \gg \frac{L N}{|A|}, \quad x_{0}^{-1} A_{x_{0}} \subset D \quad \text { and } \quad y_{0}^{-1} B_{y_{0}} \subset D^{-1} \text {. }
$$

Moreover, there exists a further subset $\tilde{A}_{x_{0}} \subseteq A_{x_{0}}$, with

$$
\left|\tilde{A}_{x_{0}}\right| \gg \frac{L M}{|A|^{3}}
$$

such that for all $z \in \tilde{A}_{x_{0}}$, letting $S_{z}=P_{z / x_{0}} \cap B_{y_{0}}$, we have

$$
\left|S_{z}\right| \gg \frac{L M N}{|A|^{4}}
$$

We require the following corollary of Lemma 11 throughout the remainder of the proof.

Claim 16. For $n \leqslant 4$ let $a_{1}, \ldots, a_{n}$ denote arbitrary elements of $\tilde{A}_{x_{0}}$. Given any set $C \subset A+1$, there exists a subset $C^{\prime} \subset C$, with $\left|C^{\prime}\right| \approx|C|$, such that the sets $a_{i} C^{\prime}$ can each be covered by

$$
O\left(\frac{|A(A+1)|^{4}}{|C||A| N^{2}}\right)
$$

translates of $\pm x_{0} A$.

Suppose $b_{1}, \ldots, b_{4} \in B_{y_{0}}$. Let

$$
\Gamma:=\frac{|A|^{2}|A(A+1)|^{4}}{M^{2}} .
$$

There exists a subset $A^{\prime} \subseteq \tilde{A}_{x_{0}}$, with $\left|A^{\prime}\right| \approx\left|\tilde{A}_{x_{0}}\right|$, such that for $1 \leqslant i \leqslant 4$ the sets $b_{i} A^{\prime}$ can each be covered by $O(\Gamma)$ translates of $\pm y_{0} A$.

Proof. We apply Lemma 11, with $X=a_{i} C, Y=a_{i} P_{a_{i} / x_{0}}, Z=A, x=a_{i}, y=a_{i}$ and $0<\epsilon<1 / 16$. Then there exist sets $C_{a_{i}} \subseteq C$ with $\left|C_{a_{i}}\right| \geqslant(1-\epsilon)|C|$ such that each of $a_{i} C_{a_{i}}$ can be covered by

$$
O_{\epsilon}\left(\frac{|A(A+1)|^{2}|A / A|}{|C|\left|a_{i} P_{a_{i} / x_{0}}\right|^{2}}\right)
$$


translates of $a_{i} P_{a_{i} / x_{0}} \subset x_{0} A$ and by at most as many translates of $-x_{0} A$. Let $C^{\prime}=$ $C_{a_{1}} \cap \cdots \cap C_{a_{n}}$, so that $\left|C^{\prime}\right| \geqslant(1-n \epsilon)|C| \geqslant(3 / 4)|C|$. Then, by (9) and (21), it follows that (25) denotes the number of translates of $\pm x_{0} A$ required to cover the sets $a_{i} C^{\prime}$ for $1 \leqslant i \leqslant n$.

Next, we apply Lemma 11, with $X=b_{i} \tilde{A}_{x_{0}}, Y=b_{i} Q_{b_{i} / y_{0}}, Z=A, x=b_{i}$ and $y=0$. Recalling (21), (22), (23) and proceeding similarly as above, we can identify a subset $A^{\prime} \subseteq \tilde{A}_{x_{0}}$, with $\left|A^{\prime}\right| \approx\left|\tilde{A}_{x_{0}}\right|$, such that the sets $b_{i} A^{\prime}$ are each fully contained in $O(\Gamma)$ translates of $\pm y_{0} A$.

We split the proof into four cases based on the nature of the quotient set $R\left(\tilde{A}_{x_{0}}\right)$.

Case 1: $R\left(\tilde{A}_{x_{0}}\right) \neq R\left(B_{y_{0}}\right)$.

Case 1.1: There exist elements $a, b, c, d \in \tilde{A}_{x_{0}}$ such that

$$
r=\frac{a-b}{c-d} \in R\left(\tilde{A}_{x_{0}}\right) \backslash R\left(B_{y_{0}}\right) .
$$

By Lemma 3, for any subset $Y \subseteq B_{y_{0}}$ with $|Y| \approx\left|B_{y_{0}}\right|$, we have

$$
\left|B_{y_{0}}\right|^{2} \approx|Y|^{2}=|Y-r Y| \leqslant|a Y-b Y-c Y+d Y| .
$$

By Claim 16 and (22), there exists a subset $B^{\prime} \subseteq B_{y_{0}}$, with $\left|B^{\prime}\right| \approx\left|B_{y_{0}}\right|$, such that $d B^{\prime}$ is contained in

$$
O\left(\frac{|A(A+1)|^{4}}{L N^{3}}\right)
$$

translates of $-x_{0} A$ and $a B^{\prime}, b B^{\prime}, c B^{\prime}$ are contained in at most the same number of translates of $x_{0} A$. Thus, setting $Y=B^{\prime}$, by $(27)$, we have

$$
\left(\frac{L N}{|A|}\right)^{2} \ll|A-A-A-A|\left(\frac{|A(A+1)|^{4}}{L N^{3}}\right)^{4} .
$$

Then, by (18), we get

$$
M^{6} N^{2}|A|^{21} \ll|A(A+1)|^{40} .
$$

By (19) and (20), we conclude the inequality

$$
|A(A+1)|^{48} \gg(\log |A|)^{-8}|A|^{49} .
$$

Case 1.2: There exist elements $a, b, c, d \in B_{y_{0}}$ such that

$$
r=\frac{a-b}{c-d} \in R\left(B_{y_{0}}\right) \backslash R\left(\tilde{A}_{x_{0}}\right) .
$$

Then for any subset $Y \subseteq \tilde{A}_{x_{0}}$ with $|Y| \approx\left|\tilde{A}_{x_{0}}\right|$, by Lemma 3, we have

$$
\left|\tilde{A}_{x_{0}}\right|^{2} \approx|Y|^{2}=|Y-r Y| \leqslant|a Y-b Y-c Y+d Y| .
$$


By the second part of Claim 16 , there exists a subset $A^{\prime} \subset \tilde{A}_{x_{0}}$, with $\left|A^{\prime}\right| \approx\left|\tilde{A}_{x_{0}}\right|$, such that the sets $a A^{\prime}, b A^{\prime}$ and $c A^{\prime}$ are each fully contained in $O(\Gamma)$ translates of $y_{0} A$ and $d A^{\prime}$ can be covered by $O(\Gamma)$ translates of $-y_{0} A$. Thus, setting $Y=A^{\prime}$, by (28), we have

$$
\left(\frac{L M}{|A|^{3}}\right)^{2} \ll|A-A-A-A|\left(\frac{|A|^{2}|A(A+1)|^{4}}{M^{2}}\right)^{4} .
$$

Applying (18) yields

$$
M^{10} L^{2}|A|^{9} \ll|A(A+1)|^{40} .
$$

Hence, by (19) and (20), we get

$$
|A(A+1)|^{52} \gg(\log |A|)^{-12}|A|^{53} .
$$

Case 2: $1+R\left(\tilde{A}_{x_{0}}\right) \nsubseteq R\left(\tilde{A}_{x_{0}}\right)$. There exist elements $a, b, c, d \in \tilde{A}_{x_{0}}$ such that

$$
r=1+\frac{a-b}{c-d} \notin R\left(\tilde{A}_{x_{0}}\right)=R\left(B_{y_{0}}\right) .
$$

Let $Y_{1} \subseteq B_{y_{0}}$ and $Y_{2} \subseteq S_{a}$ be any sets with $\left|Y_{1}\right| \approx\left|B_{y_{0}}\right|$ and $\left|Y_{2}\right| \approx\left|S_{a}\right|$. By Lemma 10, with $X=(c-d) Y_{1}$, there exists a subset $Y_{1}^{\prime} \subseteq Y_{1}$, with $\left|Y_{1}^{\prime}\right| \approx\left|Y_{1}\right|$, such that

$$
\begin{aligned}
\left|Y_{1}^{\prime}-r Y_{2}\right| & \leqslant\left|(c-d) Y_{1}^{\prime}-(c-d) Y_{2}-(a-b) Y_{2}\right| \\
& \ll \frac{\left|Y_{1}-Y_{2}\right|}{\left|Y_{1}\right|}\left|(c-d) Y_{1}-(a-b) Y_{2}\right| .
\end{aligned}
$$

Recall that $Y_{1}^{\prime} \subseteq B_{y_{0}}$ and $Y_{2} \subseteq S_{a} \subseteq B_{y_{0}}$. Then Lemma 3 gives

$$
\left|Y_{1}^{\prime}\right|\left|Y_{2}\right|=\left|Y_{1}^{\prime}-r Y_{2}\right| \text {. }
$$

Thus, by (29) we have

$$
\left|Y_{1}^{\prime}\right|\left|Y_{1}\right|\left|Y_{2}\right| \ll\left|Y_{1}-Y_{2}\right|\left|c Y_{1}-d Y_{1}-a Y_{2}+b Y_{2}\right| .
$$

Since $Y_{1}, Y_{2} \subseteq B_{y_{0}} \subseteq A+1$, we have

$$
\left|Y_{1}-Y_{2}\right| \leqslant|A-A|
$$

Recall that $\left|Y_{1}^{\prime}\right| \approx\left|Y_{1}\right| \approx\left|B_{y_{0}}\right|$ and $\left|Y_{2}\right| \approx\left|S_{a}\right|$. Then by (22), (24) and noting that $a Y_{2} \subseteq x_{0} A$, we have

$$
\left(\frac{L N}{|A|}\right)^{2}\left(\frac{L M N}{|A|^{4}}\right) \ll|A-A|\left|c Y_{1}-d Y_{1}-x_{0} A+b Y_{2}\right| .
$$

Now, by Claim 16, there exist positively proportioned subsets $B_{y_{0}}^{\prime} \subseteq B_{y_{0}}$ and $S_{a}^{\prime} \subseteq S_{a}$ such that $c B_{y_{0}}^{\prime}$ and $d B_{y_{0}}^{\prime}$ can be covered by

$$
O\left(\frac{|A(A+1)|^{4}}{L N^{3}}\right)
$$


translates of $x_{0} A$ and $b S_{a}^{\prime}$ can be covered by

$$
O\left(\frac{|A|^{3}|A(A+1)|^{4}}{L M N^{3}}\right)
$$

translates of $-x_{0} A$. Thus, setting $Y_{1}=B_{y_{0}}^{\prime}$ and $Y_{2}=S_{a}^{\prime}$, by (31) it follows that

$$
\left(\frac{L N}{|A|}\right)^{2}\left(\frac{L M N}{|A|^{4}}\right) \ll|A-A||A-A-A-A|\left(\frac{|A|^{3}|A(A+1)|^{4}}{L M N^{3}}\right)\left(\frac{|A(A+1)|^{4}}{L N^{3}}\right)^{2} .
$$

Using (17) and (18), this is further reduced to

$$
M^{8}|A|^{21} \ll|A(A+1)|^{44} .
$$

Thus, by (19), we get

$$
|A(A+1)|^{52} \gg(\log |A|)^{-8}|A|^{53} .
$$

Case 3: $x_{0}^{-1} \tilde{A}_{x_{0}} \cdot R\left(\tilde{A}_{x_{0}}\right) \nsubseteq R\left(\tilde{A}_{x_{0}}\right)$. There exist elements $a, b, c, d, e \in \tilde{A}_{x_{0}}$ such that

$$
r=\frac{a}{x_{0}} \frac{b-c}{d-e} \notin R\left(\tilde{A}_{x_{0}}\right)=R\left(B_{y_{0}}\right) .
$$

Given any set $Y_{1} \subseteq B_{y_{0}}$, recalling that $S_{a} \subseteq B_{y_{0}}$, it follows from Lemma 3 that

$$
\left|Y_{1}\right|\left|S_{a}\right|=\left|Y_{1}-r S_{a}\right|
$$

For an arbitrary set $Y_{2}$, we apply Lemma 9 , with $X=\frac{b-c}{d-e} Y_{2}$, to get

$$
\begin{aligned}
\left|Y_{2}\right|\left|Y_{1}\right|\left|S_{a}\right| & =\left|Y_{2}\right|\left|Y_{1}-r S_{a}\right| \\
& \leqslant\left|Y_{1}+\frac{b-c}{d-e} Y_{2}\right|\left|Y_{2}-\frac{a}{x_{0}} S_{a}\right| \\
& \leqslant\left|d Y_{1}-e Y_{1}+b Y_{2}-c Y_{2}\right|\left|Y_{2}-A\right| .
\end{aligned}
$$

By Claim 16, we can identify sets $C_{1} \subseteq S_{d}$ and $C_{2} \subseteq P_{c / x_{0}}$ with $\left|C_{1}\right| \approx\left|S_{d}\right|$ and $\left|C_{2}\right| \approx\left|P_{c / x_{0}}\right| \approx N$, such that $e C_{1}$ is covered by

$$
O\left(\frac{|A|^{3}|A(A+1)|^{4}}{L M N^{3}}\right)
$$

translates of $x_{0} A$ and $b C_{2}$ is covered by

$$
O\left(\frac{|A(A+1)|^{4}}{|A| N^{3}}\right)
$$

translates of $-x_{0} A$. We set $Y_{1}=C_{1}$ and $Y_{2}=C_{2}$. Then, by (21), (24) and particularly noting that $d Y_{1}, c Y_{2} \subset x_{0} A$ and $Y_{2} \subset A+1$, we have

$$
N\left(\frac{L M N}{|A|^{4}}\right)^{2} \ll|A-A \| A-A-A-A|\left(\frac{|A|^{3}|A(A+1)|^{4}}{L M N^{3}}\right)\left(\frac{|A(A+1)|^{4}}{|A| N^{3}}\right) .
$$


Using (17) and (18) we get

$$
M^{6} N^{3}|A|^{20} \ll|A(A+1)|^{40} .
$$

By (19) and (20), we conclude

$$
|A(A+1)|^{49} \gg(\log |A|)^{-9}|A|^{50} .
$$

Case 4: Suppose that Cases 1-3 do not happen. Observing that $R\left(x_{0}^{-1} \tilde{A}_{x_{0}}\right)=R\left(\tilde{A}_{x_{0}}\right)$, by Lemma 5 we deduce that $R\left(\tilde{A}_{x_{0}}\right)$ is the field generated by $x_{0}^{-1} \tilde{A}_{x_{0}}$. Then according to the assumptions of Theorem 1 , we consider the following three cases.

Case 4.1: $R\left(\tilde{A}_{x_{0}}\right)=\mathbb{F}_{q}$ and $\left|\tilde{A}_{x_{0}}\right|>q^{1 / 2}$. Let $Y$ denote an arbitrary subset of $\tilde{A}_{x_{0}}$ with $|Y| \approx\left|\tilde{A}_{x_{0}}\right|$. By Lemma 7 , there exists an element $\xi \in \mathbb{F}_{q}^{*}$ such that $q \ll|Y+\xi Y|$. Since $R\left(B_{y_{0}}\right)=R\left(\tilde{A}_{x_{0}}\right)=\mathbb{F}_{q}$, there exist elements $a, b, c, d \in B_{y_{0}}$, such that

$$
q \ll|a Y-b Y+c Y-d Y| .
$$

By Claim 16, we can identify a positively proportioned subset $A^{\prime} \subset \tilde{A}_{x_{0}}$, such that $a A^{\prime}$, $b A^{\prime}$ and $d A^{\prime}$ can be covered by $O(\Gamma)$ translates of $y_{0} A$ and $c A^{\prime}$ can be covered by $O(\Gamma)$ translates of $-y_{0} A$. Thus, setting $Y=A^{\prime}$, we have

$$
q \ll|A-A-A-A|\left(\frac{|A|^{2}|A(A+1)|^{4}}{M^{2}}\right)^{4} .
$$

By (18), we get

$$
M^{8}|A|^{15} q \ll|A(A+1)|^{40} .
$$

By (19), this gives the bound

$$
|A(A+1)|^{48} \gg q(\log |A|)^{-8}|A|^{47} .
$$

We point out that if $\left|\tilde{A}_{x_{0}}\right|>q^{1 / 2}$ then one only needs to consider Cases 1.1 and 4.1 , since by Lemma 4 we have $R\left(\tilde{A}_{x_{0}}\right)=\mathbb{F}_{q}$.

Case 4.2: Either $R\left(\tilde{A}_{x_{0}}\right)=\mathbb{F}_{q}$ and $\left|\tilde{A}_{x_{0}}\right| \leqslant q^{1 / 2}$ or $R\left(\tilde{A}_{x_{0}}\right)$ is a proper subfield and $\left|A \cap c R\left(\tilde{A}_{x_{0}}\right)\right| \ll\left|R\left(\tilde{A}_{x_{0}}\right)\right|^{1 / 2}$ for all $c \in \mathbb{F}_{q}$. Since $R\left(\tilde{A}_{x_{0}}\right)$ is the field generated by $x_{0}^{-1} \tilde{A}_{x_{0}}$, we have $\tilde{A}_{x_{0}} \subseteq x_{0} R\left(\tilde{A}_{x_{0}}\right)$. Hence

$$
\left|\tilde{A}_{x_{0}}\right|^{2}=\left|\tilde{A}_{x_{0}} \cap x_{0} R\left(\tilde{A}_{x_{0}}\right)\right|^{2} \leqslant\left|A \cap x_{0} R\left(\tilde{A}_{x_{0}}\right)\right|^{2} \ll\left|R\left(\tilde{A}_{x_{0}}\right)\right| .
$$

Now, recalling that $R\left(\tilde{A}_{x_{0}}\right)=R\left(B_{y_{0}}\right)$, by Lemma 6 , there exist elements $a, b, c, d \in B_{y_{0}}$ such that for any subset $Y \subseteq \tilde{A}_{x_{0}}$ with $|Y| \approx\left|\tilde{A}_{x_{0}}\right|$, we have

$$
|Y|^{2} \ll|a Y-b Y+c Y-d Y| \text {. }
$$

By Claim 16, there exists a subset $A^{\prime} \subseteq \tilde{A}_{x_{0}}$, with $\left|A^{\prime}\right| \approx\left|\tilde{A}_{x_{0}}\right|$, such that $c A^{\prime}$ can be covered by $O(\Gamma)$ translates of $-y_{0} A$ and $a A^{\prime}, b A^{\prime}, d A^{\prime}$ can be covered by $O(\Gamma)$ translates of $y_{0} A$. We set $Y=A^{\prime}$ so that, by (32), we obtain

$$
\left(\frac{L M}{|A|^{3}}\right)^{2} \ll|A-A-A-A|\left(\frac{|A|^{2}|A(A+1)|^{4}}{M^{2}}\right)^{4} .
$$


Applying (18) gives

$$
M^{10} L^{2}|A|^{9} \ll|A(A+1)|^{40} .
$$

Then, by (19) and (20), we have

$$
|A(A+1)|^{52} \gg(\log |A|)^{-12}|A|^{53} .
$$

Case 4.3: $R\left(\tilde{A}_{x_{0}}\right)$ is a proper subfield and $\left|A \cap x_{0} R\left(\tilde{A}_{x_{0}}\right)\right| \ll|A|^{25 / 26}$. Recall that $\tilde{A}_{x_{0}} \subset x_{0} R\left(\tilde{A}_{x_{0}}\right)$. Then, by (23) and (20), we get

$$
\frac{M^{2}}{|A|^{5}} \ll\left|\tilde{A}_{x_{0}}\right| \ll|A|^{25 / 26} .
$$

Using (19), we recover the bound

$$
|A(A+1)|^{52} \gg(\log |A|)^{-52}|A|^{53} .
$$

\section{Proof of Corollary 2}

Let $\alpha \in \mathbb{F}_{q}^{*}$ and denote $S=A \cap(A-\alpha)$. Observing that $S, S+\alpha \subset A$, we deduce $|S(S+\alpha)| \leqslant|A A|$. Then, estimate (8) follows by applying Theorem 1 to the set $S$. Now, since $S \subset A$, if $A$ satisfies restriction (7), then $S$ can fail to satisfy restriction (6) only if $|S| \ll|A A|^{52 / 53}$, which in fact gives the required estimate. This concludes the proof of estimate (8).

Next, noting that

$$
|A \cap(A-\alpha)|=\left|\left\{\left(a_{1}, a_{2}\right) \in A^{2}: a_{1}-a_{2}=\alpha\right\}\right|,
$$

similarly to (10) and (11), we have the identities

$$
|A|^{2}=\sum_{\alpha \in A-A}|A \cap(A-\alpha)| \text { and } E_{+}(A)=\sum_{\alpha \in A-A}|A \cap(A-\alpha)|^{2} .
$$

In particular, it follows that

$$
E_{+}(A) \ll|A|^{2} \cdot \max _{\alpha \in \mathbb{F}_{q}^{*}}|A \cap(A-\alpha)| .
$$

Thus the required bound on $E_{+}(A)$ follows from (8).

\section{Acknowledgements}

The author would like to thank Igor Shparlinski for helpful conversations. The author also gratefully acknowledges the financial support provided by IPM during the preparation of this paper. 


\section{References}

[1] J. Bourgain and A. Glibichuk. Exponential sum estimates over a subgroup in an arbitrary finite field. J. Anal. Math., 115(1):51-70, 2011.

[2] M. Z. Garaev and C.-Y. Shen. On the size of the set $A(A+1)$. Mathematische Zeitschrift, 265(1):125-132, 2010.

[3] T. G. F. Jones. Explicit incidence bounds over general finite fields. Acta Arith., 150(3):241-262, 2011.

[4] T. G. F. Jones and O. Roche-Newton. Improved bounds on the set $A(A+1)$. J. Combin. Theory, Ser. A., 120(3):515-526, 2013.

[5] N. H. Katz and C.-Y. Shen. A slight improvement to Garaev's sum product estimate. Proc. Amer. Math. Soc., 136(7):2499-2504, 2008.

[6] L. Li and O. Roche-Newton. An improved sum-product estimate for general finite fields. SIAM J. Discrete Math., 25(3):1285-1296, 2011.

[7] M. Rudnev. An improved sum-product inequality in fields of prime order. Int. Math. Res. Not. IMRN, 16:3693-3705, 2012.

[8] M. Rudnev. On the number of incidences between planes and points in three dimensions. Combinatorica, 38(1):219-254, 2018.

[9] S. Stevens and F. de Zeeuw. An improved point-line incidence bound over arbitrary fields. Bull. London Math. Soc., 49(5):842-858, 2017.

[10] T. Tao and V. Vu. Additive combinatorics. Cambridge Univ. Press, 2006.

[11] A. Warren. On products of shifts in arbitrary fields. Mosc. J. Comb. Number Theory, 8(3):247-261, 2019.

[12] D. Zhelezov. On additive shifts of multiplicative almost-subgroups in finite fields. arXiv: 1507.05548 (to appear in Proc. Amer. Math. Soc.). 